\title{
Ice bucket challenge bears fruit for amyotrophic lateral sclerosis
}

\author{
James Hrastelj $^{1} \cdot$ Neil P. Robertson ${ }^{1}$
}

Published online: 12 October 2016

(C) The Author(s) 2016. This article is published with open access at Springerlink.com

\section{Introduction}

In 2014, the 'ice bucket challenge' raised over $\$ 140$ million worldwide for research into amyotrophic lateral sclerosis (ALS). This unprecedented boost to research funding is now beginning to deliver significant advances in our understanding of the pathogenesis of this devastating disease, and all the studies in this month's journal club were, at least in part, funded by charitable organisations that benefited from these monies.

The first paper we discuss reports the results of the largest genetic study of ALS to date, with discovery of three new genes associated with ALS by utilising novel whole-genome sequencing technology. Importantly, the study also illuminates the genetic architecture of ALS, which in turn is likely to inform design of future genetic studies and suggests opportunities for clinical research to establish genotype-phenotype correlations. However, as genetic studies of rapidly increasing power reveal increasing numbers of risk variants and mutations, the next substantial challenge is to understand how such variants cause disease. The second and third papers discussed this month report on attempts to understand how two leading ALS risk variants cause disease and may provide exciting avenues for the development of novel therapeutic agents. The association of the C9orf72 repeat expansion with ALS, reviewed in journal club in 2012 and 2015, is now well documented and Kramer et al. report notable progress in translating our understanding into novel therapeutic targets.

Neil P. Robertson

robertsonnp@cardiff.ac.uk

1 Institute of Psychological Medicine and Clinical, Neurosciences, Cardiff University, Cardiff CF14 4XW, UK
Similarly, Ito et al. report that mutations in the established ALS risk gene optineurin may function by sensitising cells to a form of regulated cell death, which could again be targeted for therapeutic intervention.

\section{Genome-wide association analyses identify new risk variants and the genetic architecture of amyotrophic lateral sclerosis}

Genetic sequencing technology is advancing at a considerable pace, and learning how to effectively harness the power of whole-genome sequencing will provide information of unparalleled depth and detail. Van Rheenen et al. used whole-genome sequencing data on 1246 ALS cases and 615 controls to dramatically improve the sensitivity of genetic screening to enable detection of low-frequency variants by imputation. An impressive 8.7 million SNPs were genotyped in 7763 ALS cases and 4669 controls, and by combining with genotyping data from 41 other genetic studies of ALS, a meta-analysis was performed on a total of 12,577 ALS cases and 23,475 controls. The study reports three new independent loci that associate with ALS risk with genome-wide significance: C27orf2, MOBP and $S C F D 1$, which were all replicated in subsequent analysis of 2579 ALS cases and 2767 controls. Three previously associated loci were also replicated: C9orf72, SARM1 and UNC13A.

The authors go on to report fine mapping and functional data that begin to explain how the newly discovered variants confer risk to ALS. Fine mapping of C27orf2 in 2562 ALS cases and 1138 controls demonstrated an excess of loss-of-function mutations, suggesting pathology is caused by reduced protein product of C27orf2. Also, publicly available gene expression data from human brain were 
used to search for SNPs at the risk loci that correlate with expression of nearby genes. This analysis revealed that the variants at SARM1 and UNC13A appear to, at least partly, influence function by controlling expression of the genes POLDIP2 and KCNN1, respectively.

Finally, the study proceeded to analyse the genetic architecture of ALS by estimating the proportion of heritability explained by low versus high frequency variants. When compared with schizophrenia, a classical polygenic disorder dominated by common variants, the genetic risk for ALS appears to be dominated by a large number of rare variants that each confer substantial risk.

Comment This powerful study identifies three new loci that confer risk to ALS and provides some initial information on the possible pathogenic mechanisms. This is by far the largest genetic study in ALS to date and goes some way to resolving a fundamental question about the genetic architecture of ALS. Complementary to this work is a study published in the same edition of Nature Genetics by Kenna et al. who report discovery of a new gene implicated in familial ALS by exome sequencing. As a substantial proportion of the heritability of ALS is conferred by a large number of rare variants, each conferring relatively high risk to disease, future genetic studies of ALS must ensure that rare variants are captured. Such a genetic architecture implies that clinicians will have a key role in establishing genotype-phenotype correlations, which will help determine how genetic factors contribute to clinical heterogeneity.

Van Rheenen W et al. (2016) Nature Genetics 48(9):1043-1046.

\section{Spt4 selectively regulates the expression of $C 9$ orf 72 sense and antisense mutant transcripts}

Expansions of the hexanucleotide repeat within the C9orf72 gene are the leading genetic cause of ALS, being responsible for 7-10\% cases of sporadic ALS and $40 \%$ of familial cases. In a previous journal club earlier this year, we discussed a paper that explored the effect of regulation of $C 9$ orf72 on clinical outcomes, which added to the data suggesting that the expansion caused a pathological gainof-function. Kramer and colleagues go further in this paper by identifying a potential therapeutic avenue based on reducing the expression of C9orf72. Work in Huntington's disease (HD) and on C9orf72 suggests two major mechanisms by which such expansions can cause disease: RNA toxicity by aggregation of RNA transcribed from the repeats into intracellular foci that disrupt cellular machinery, and protein toxicity by aberrant translation of the repeat mRNA into toxic dipeptide repeat proteins (DPRs).
The authors hypothesise that $S p t 4$, a transcription factor recently discovered to mediate transcription of long repeat sequences in HD, could be involved in transcription of the pathological hexanucleotide repeat expansion in C9orf 72 . The study generated three models with the pathological expansion (yeast, C. elegans and Drosophila) and found that deleting or knocking down Spt4 reduced or eradicated RNA foci aggregation and DPR production.

The experiment was repeated in human fibroblasts isolated from ALS cases with (c9ALS) and without the C9orf72 repeat expansion and healthy controls and detected foci of sense and antisense expansion RNA strands and DPR proteins exclusively in c9ALS fibroblasts. Using RNA interference to knock down the mammalian version of Spt4 resulted in decreased C9orf72 mRNA and PDR proteins, and reduced sense and antisense RNA foci. This effect was partially replicated in human cortical neurons generated from induced pluripotent stem cells.

Comment This study illustrates how results from genetic studies can begin to be translated into therapeutic targets. Whilst the mechanism of toxicity of the C9orf72 expansion has not been confirmed, it is likely that treatments capable of reducing its expression will be beneficial. Targeting transcription apparatus can produce far-reaching effects on other genes, but this study also reported that the effect of reducing the expression of Spt4 had minimal effects on the expression of other genes using RNA sequencing, suggesting that this could be a relatively specific therapeutic target.

Kramer NJ et al. (2016) Science 353(6300):708-12.

\section{RIPK1 mediates axonal degeneration by promoting inflammation and necroptosis in ALS}

Optineurin is a gene that has an established association with familial ALS, but the pathogenic mechanism is unclear. In this paper, Ito et al. report that one mechanism appears to be sensitising cells to a form of regulated cell death called necroptosis through the activity of the enzyme RIPK1.

The authors generated a mouse model with homozygous deletions of optineurin, which developed a progressive degenerative motor neuropathy and spinal cord histopathology similar to ALS. Mice that were also generated to be homozygous for inactivated $R I P K 1$ displayed reversed spinal cord pathology and motor dysfunction.

Several different types of mouse cells including embryonic fibroblasts, adipose cells, cultured oligodendrocytes and spinal cord samples were subject to optineurin deletions or knockdowns and displayed evidence of 
increased mediators of necroptosis including RIPKI. Interestingly, cultured oligodendrocytes homozygous for optineurin deletions underwent significantly greater cell death and this was reversed in cells also homozygous for RIPK1 deletions or by giving an inhibitor of necroptosis (Nec-1s). In addition, microglia homozygous for optineurin deletions had increased levels of RIPKI activation and acquired a proinflammatory phenotype.

The study then investigated whether the mechanism is generalisable by investigating the SODl mouse model. Markers of necroptosis were found to be raised in spinal cord samples and the mouse displayed a similar motor deficit, and both were reversed by administration of Nec1s. Finally, analysis of spinal cord samples from human sporadic ALS cases revealed increased markers of necroptosis in microglia and oligodendrocytes.

Comment By investigating the functional consequences of mutations of an established risk gene, this work provides the first evidence that necroptosis may be an important pathological mechanism in ALS that could be targeted for therapeutic intervention. Interestingly, the mechanism appears to be more important in oligodendrocytes and microglia rather than neurons, which suggests that cells other than neurons may mediate at least part of the neurodegenerative process. Before pursuing potential therapeutic agents targeting necroptosis, however, further work in human cells is necessary to determine its role in neurodegeneration in familial and sporadic forms of ALS.

Ito $\mathrm{Y}$ et al. (2016) Science 353(6299):603-8.

Open Access This article is distributed under the terms of the Creative Commons Attribution 4.0 International License (http://crea tivecommons.org/licenses/by/4.0/), which permits unrestricted use, distribution, and reproduction in any medium, provided you give appropriate credit to the original author(s) and the source, provide a link to the Creative Commons license, and indicate if changes were made. 